\title{
Actualisation des connaissances sur l'abeille Megachile sculpturalis SMITH, 1853 en France et en Europe (Hymenoptera : Megachilidae)
}

\author{
Violette LE FÉON ${ }^{1}$ (D) . David GeNOUD ${ }^{2}$ (D) - Benoît GESLIN ${ }^{3}$ (D)
}

LE FÉON, V., D. GenOUd \& B. GESLIN (2021). Actualisation des connaissances sur l'abeille Megachile sculpturalis SMITH, 1853 en France et en Europe (Hymenoptera : Megachilidae). Osmia, 9: 25-36. https://doi.org/10.47446/OSMIA9.4

\begin{abstract}
Résumé
Megachile sculpturalis est une abeille originaire de l'est de l'Asie aujourd'hui également présente en Amérique du Nord et en Europe. Cet article fait suite à la première synthèse sur l'écologie et la distribution géographique en Europe de cette espèce parue dans cette même revue en 2018 et propose une actualisation des connaissances. Megachile sculpturalis a été observée dans 13 pays européens. Son aire de répartition s'étend aujourd'hui des côtes atlantiques françaises à l'ouest à la péninsule de Crimée à l'est. Elle a par ailleurs été signalée dans deux îles méditerranéennes, lîle d'Elbe (Italie) et Majorque (Espagne). En France, entre 2008 et 2020, elle a été observée dans 259 communes réparties dans 44 départements. L'observation française la plus septentrionale a été réalisée en 2020 près de Reims dans la Marne. Concernant le comportement alimentaire, de nouvelles analyses polliniques viennent corroborer les résultats de précédentes études qui suggéraient un régime polylectique avec une préférence marquée pour les arbustes d'origine asiatique (Sophora japonica et Ligustrum sp. en particulier). Pour sa nidification, M. sculpturalis utilise massivement les hôtels à abeilles. Cependant, une gamme de diamètres (trous dans des bûches ou sections de tiges végétales) compris entre 4 et $8 \mathrm{~mm}$ permet d'héberger les principales espèces indigènes susceptibles de nidifier dans les hôtels, tout en limitant l'installation de M. sculpturalis. De nouvelles observations ont confirmé la tendance de M. sculpturalis à vider les nids d'autres espèces pour y installer ses propres cellules larvaires. Par ailleurs, en Suisse, une femelle a été observée tuant une abeille du genre Heriades. Ce type d'événements, s'ils s'avèrent fréquents, pourraient avoir des impacts négatifs sur les abeilles indigènes mais les données sont pour l'heure insuffisantes pour quantifier ces impacts sur les populations.
\end{abstract}

Mots-clefs | espèce exotique $\cdot$ distribution géographique $\cdot$ impacts écologiques $\cdot$ nidification $\cdot$ régime alimentaire $\cdot$ sciences participatives

Update of knowledge on the bee Megachile sculpturalis SMITH, 1853 in France and Europe (Hymenoptera: Megachilidae)

\begin{abstract}
Megachile sculpturalis is a bee native to eastern Asia that is now also present in North America and Europe. This article follows the first synthesis on the ecology and geographical distribution in Europe of this species published in this same journal in 2018 and updates the knowledge. Megachile sculpturalis has been observed in 13 European countries. Its range now extends from the French Atlantic coast in the west to the Crimean Peninsula in the east. It has also been recorded on two Mediterranean islands, Elba (Italy) and Mallorca (Spain). In France, between 2008 and 2020, it was observed in 259 communes in 44 departments. The most northerly French observation was made in 2020 near Reims in the Marne department. Concerning feeding behaviour, new pollen analyses corroborate the results of previous ones which suggested a polylectic diet with a marked preference for shrubs of Asian origin (especially Sophora japonica and Ligustrum sp.). Regarding nesting behaviour, M. sculpturalis makes extensive use of bee hotels. However, a range of diameters (holes in logs or sections of plant stems) between 4 and $8 \mathrm{~mm}$ is suitable for the main native species likely to nest in the hotels, while limiting the establishment of M. sculpturalis. New observations have confirmed the tendency of $M$. sculpturalis to empty the nests of other species in order to establish its own larval cells. In addition, in Switzerland, a female was observed killing a bee of the genus Heriades. Such events, if frequent, could have negative impacts on native bees, but data are currently insufficient to quantify these impacts on populations.
\end{abstract}

Keywords $\mid$ exotic species $•$ geographical distribution $\cdot$ ecological impacts $\bullet$ nesting behaviour $\cdot$ diet $\bullet$ citizen science

Reçu • Received | 20 June 2021 || Accepté • Accepted | 09 July 2021 || Publié (en ligne) • Published (online) | 12 July 2021

Reviewers | T. JEAN • A. PERRARD \|| http://zoobank.org/F5F36611-44E4-4613-9848-5A71BD6B9FF6

${ }^{1}$ [VLF] 10 rue de l'Olivraie, F - 44200 Nantes, France • violette.lefeon@gmail.com

(D) https://orcid.org/0000-0002-0474-9364 • zoo:ank http://zoobank.org/36669374-8A39-4C8E-8F23-A15909469029

2 [DG] 2ter avenue des Roses, F - 87240 Ambazac, France • dge-davidgenoud@orange.fr

(iD) https://orcid.org/0000-0001-8564-040X • zoosank http://zoobank.org/B9EA26E1-94B2-491E-BE27-1ACE034C777C

${ }^{3}$ [BG] Institut Méditerranéen de Biodiversité et d'Écologie Marine et Continentale (IMBE), UMR CNRS 7263 IRD 237 (Aix-Marseille Université / Avignon Université / CNRS / IRD), Campus Sciences St Jérome, Avenue Escadrille Normandie Niémen, F -13397 Marseille cedex 2013331 •benoitgeslin@gmail.com (iD) https://orcid.org/0000-0002-2464-7998 • zoo:ank http://zoobank.org/5761C7DE-A10D-4B13-AD08-725B285BD899 


\section{INTRODUCTION}

Megachile sculpturalis (figures 1-2) est une abeille originaire de l'est de l'Asie observée pour la première fois en Europe en 2008 près de Marseille (VEREECKEN \& BARBIER, 2009). Nous présentions en 2018, dans ce même journal, un état des connaissances sur son écologie et sa distribution géographique en France et en Europe (LEFÉON\& GESLIN, 2018).

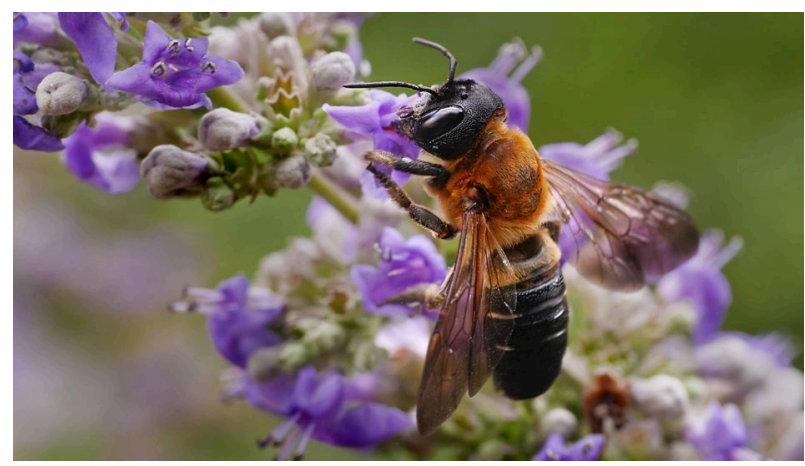

Figure 1. Femelle de Megachile sculpturalis. Cliché F. VASSEN (juillet 2016, Le Collet-de-Dèze, Lozère, France).
Nous avons depuis poursuivi le suivi de son expansion géographique en France et en présentons ici les résultats. Nous proposons également une synthèse des informations disponibles sur sa distribution en Europe et des données acquises par les études scientifiques dont l'espèce a fait l'objet ces dernières années.

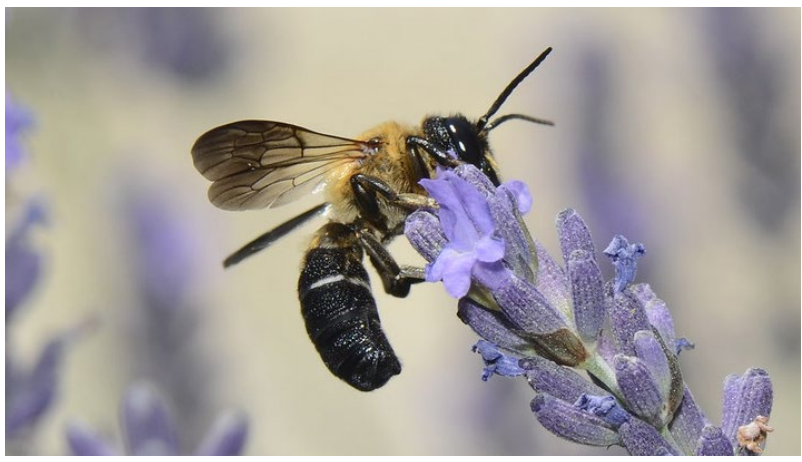

Figure 2. Mâle de Megachile sculpturalis. Cliché G. DE PRÉMOREL (juin 2014, Étoile, Drôme, France).

\section{DISTRIBUTION GÉOGRAPHIQUE EN EUROPE}

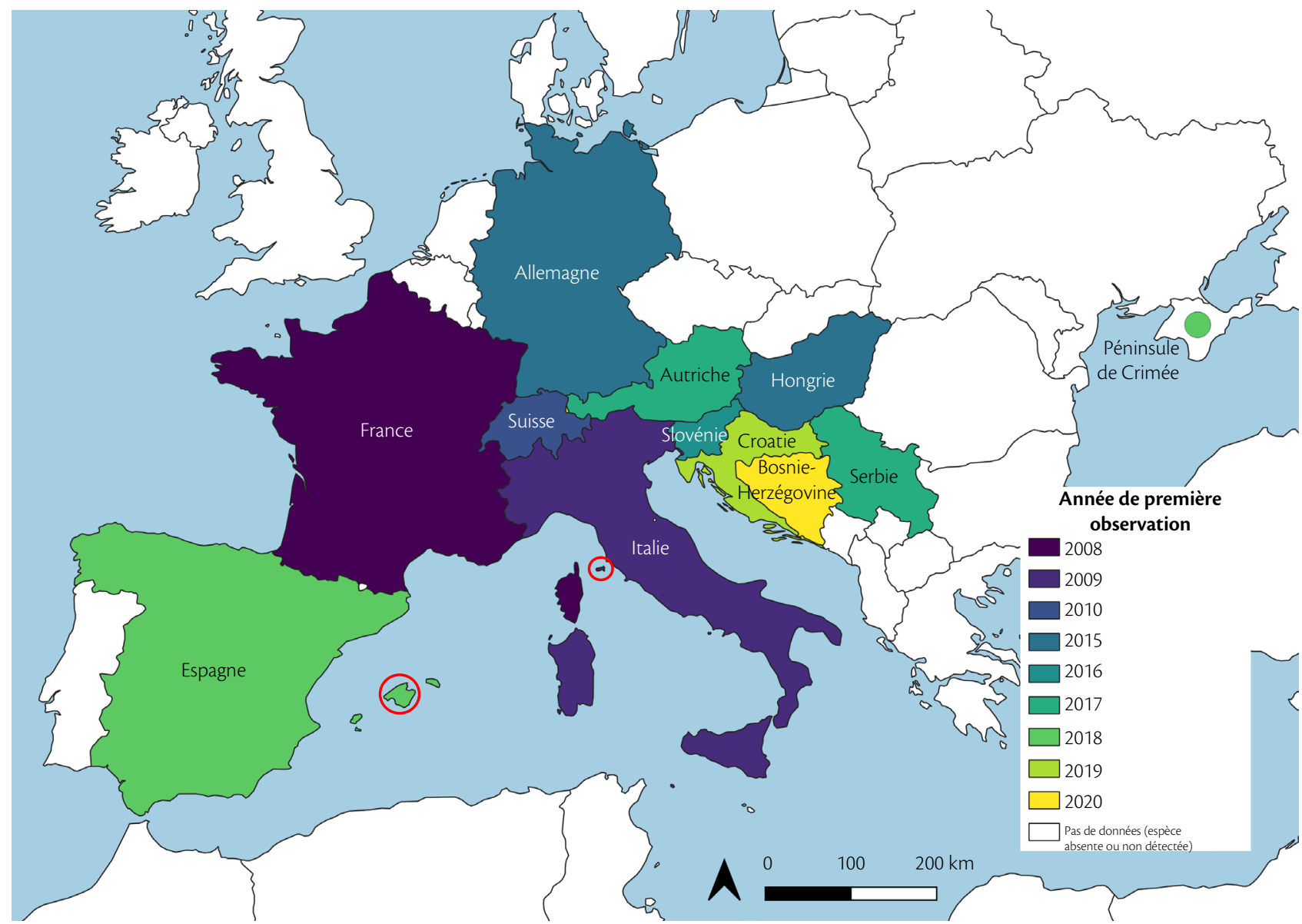

Figure 3. Année de première observation de Megachile sculpturalis dans les pays européens.

Sources : AGUADO et al., 2018 ; AMIET, 2012 ; ĆETKOVIĆ et al., 2020 ; GOGALA \& ZADRAVEC, 2018 ; IVANOV \& FATERYGA, 2019 ; KOVÁCS, 2015 ; LANNER et al., 2020 ; QUARANTA et al., 2014 ; VEREECKEN \& BARBIER, 2009 ; WESTRICH et al., 2015; WeSTRICH, 2017. La coloration homogène de l'ensemble des territoires nationaux peut être trompeuse. Ainsi, concernant les îles, M. sculpturalis est jusqu'à présent inconnue de Corse, de Sicile ou de Sardaigne. Elle a cependant été observée sur lî̂le d'Elbe et à Majorque, îles que nous avons entourées en rouge. Carte réalisée sous QGIS version 3.14, fond de carte téléchargé sur Natural Earth (https://www.naturalearthdata.com) 
Entre 2008 et 2020, M. sculpturalis a été observée dans 13 pays européens (figure 3). L'observation en France de 2008 a rapidement été suivie d'observations en Italie en 2009 (QUARANTA et al., 2014) et en Suisse en 2010 (AMIET, 2012). S'en suit une période de cinq ans au cours de laquelle elle n'est pas signalée dans d'autres pays. À partir de 2015, la liste s'allonge rapidement : $M$. sculpturalis est observée en Allemagne (WESTRICH et al., 2015) et en Hongrie (KOVÁCS, 2015) en 2015, en Slovénie en 2016 (GoGALA \& ZADRAVEC, 2018), en Autriche (WESTRICH, 2017) et en Serbie (ĆETKOVIĆ et al., 2020) en 2017, en Espagne (AGUADO et al., 2018; ORTIZSÁNCHEZ et al., 2018) et dans la péninsule de Crimée (IVANOV \& FATERYGA, 2019) en 2018, en Croatie (https://www.inaturalist.org) et au Liechtenstein (LANNER et al., 2020a) en 2019 et en Bosnie-Herzégovine en 2020 (https://www.inaturalist.org). En l'état actuel de nos connaissances, en Europe, l'aire de répartition de M. sculpturalis s'étend des côtes atlantiques françaises (commune d'Anglet) à l'ouest à la péninsule de Crimée à l'est et de Nonnweiler (Sarre, Allemagne) au nord, à Morano Calabro (Calabre, Italie) au sud.

La situation diffère bien sûr fortement selon les pays, en fonction du moment de l'arrivée de $M$. sculpturalis sur le territoire, des conditions locales et aussi probablement de la qualité du suivi et du nombre d'observateurs mobilisés. Par exemple, l'espèce est connue de 138 localités en Italie (RUZZIER et al., 2020, état des connaissances en décembre 2019) et de 28 localités en Allemagne (WESTRICH, 2020a, état des connaissances au 3 septembre 2020). À l'opposé, nous n'avons connaissance que d'une seule observation pour la
Croatie et la Bosnie-Herzégovine (données recueillies sur le site https://www.inaturalist.org).

Ces dernières années ont également été marquées par l'observation de $M$. sculpturalis sur deux îles méditerranéennes : sur l'île d'Elbe (Italie) en 2019 (RUZZIER et al., 2020) et à Majorque, plus grande île de l'archipel des Baléares (Espagne) en 2020 (RIBAS-MARQUÈs \& DÍAZ-CALAFAT, 2021).

Plusieurs programmes de sciences participatives ont été mis en place afin de collecter le maximum de données d'observations. C'est par exemple le cas en Serbie (ĆETKOVIĆ et al., 2020), en Autriche, en Suisse et au Liechtenstein (LANNER et al., 2020a). L'implication du grand public est possible dans le cas de $M$. sculpturalis car l'espèce est facilement identifiable (LE FÉON \& GESLIN, 2018). Quelques précautions sont toutefois nécessaires car d'autres abeilles, des andrènes notamment, possèdent une coloration semblable. L'idéal est donc que chaque témoignage soit accompagné d'une photographie.

Dans un article paru tout récemment, BILA DUBAIĆ \& LANNER (2021) émettent l'idée que les apiculteurs pourraient être un public à cibler particulièrement dans les programmes de sciences participatives sur M. sculpturalis. Les auteurs soulignent l'intérêt des apiculteurs pour les insectes, le temps passé à l'extérieur, leur capacité à reconnaître l'espèce et à manipuler les abeilles, lorsqu'il s'agit de collecter des spécimens pour des analyses génétiques par exemple.

\section{MÉCANISMES DE DISPERSION ET HISTORIQUE DE L'INVASION EN EUROPE}

L'expansion géographique rapide de $M$. sculpturalis résulte probablement de deux mécanismes (LANNER et al., 2020a). Tout d'abord, les caractéristiques intrinsèques de l'espèce (sa forte capacité de vol liée à sa grande taille ainsi que son régime alimentaire et son comportement de nidification, $c f$. ci-dessous) en font une colonisatrice efficace une fois introduite dans un nouvel endroit. II en résulte une dispersion à courte distance, pas à pas, aboutissant à la colonisation progressive d'une région par exemple. À cela s'ajoute la dispersion liée au transport de marchandises, bois ou autre support pouvant contenir des larves, qui peut conduire à des déplacements sur de longues distances et qui explique probablement les «bonds » de plusieurs centaines de kilomètres que semble faire l'espèce (LANNER et al., 2020a). Par ailleurs, l'analyse génétique de plusieurs populations européennes a récemment suggéré que plusieurs évènements d'introduction indépendants pouvaient également être un facteur explicatif de la progression rapide de M. sculpturalis en Europe (LANNER et al., 2021). Des individus provenant du sud et du centre de la France, de Suisse et de Vienne (Autriche) ont été génotypés. Les résultats montrent des différences génétiques importantes entre les individus provenant d'Autriche et ceux de France et de Suisse. Ce résultat suggère donc qu'au moins deux événements d'introduction ont eu lieu. D'autres études génétiques sont en cours, qui devraient notamment permettre de comprendre si les individus qui se trouvent en Europe se rapprochent génétiquement plus des populations du Sud-Est asiatique, aire de répartition originelle de l'espèce, ou des populations d'Amérique du Nord, zone où l'espèce a été introduite dès 1994 (MANGUM \& BROOKS, 1997).

\section{DISTRIBUTION GÉOGRAPHIQUE EN FRANCE}

Megachile sculpturalis a été observée pour la première fois en 2008 en France, à Allauch près de Marseille dans le département des Bouches-du-Rhône (VEREECKEN \& BARBIER, 2009).

Dix ans plus tard, un premier bilan de l'expansion géographique de l'espèce en France a été dressé pour la période allant de 2008 à 2016 (LE FÉON et al., 2018) en se basant sur les données recueillies directement auprès de naturalistes (notamment grâce à la liste de discussion Apoidea gallica) ou sur différents forums et sites dédiés à l'entomologie (Le Monde des Insectes ou le site du Spipoll Suivi photographique des insectes pollinisateurs par exemple). Ce bilan indiquait que les premières années, la présence de $M$. sculpturalis sur le territoire s'est faite discrète car aucune autre observation n'a été mentionnée. En 2011, 


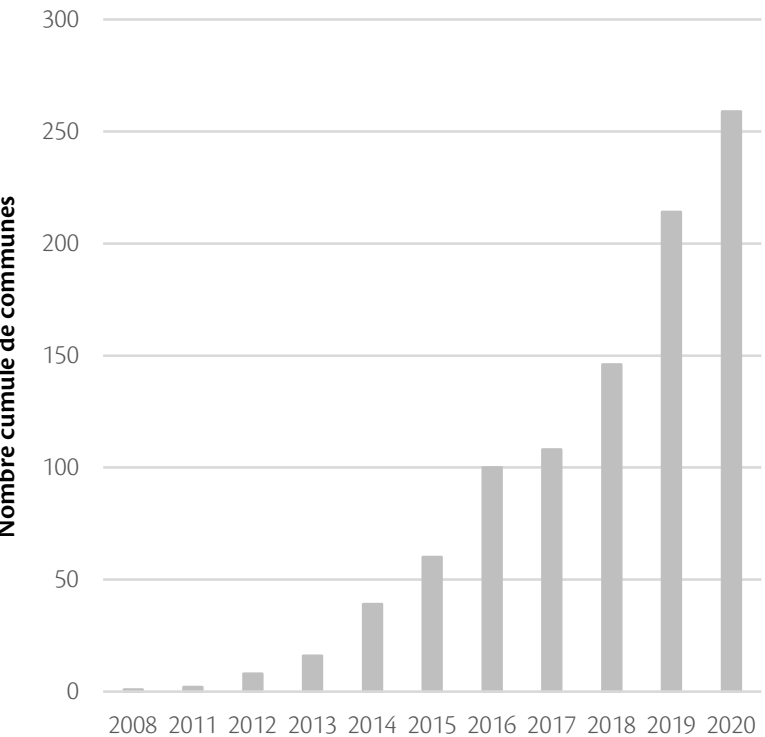

Année

Figure 4. Nombre de communes françaises, cumulé année après année, comptant une ou plusieurs observations de Megachile sculpturalis. D’après nos données, au 31 mai 2021, l'espèce a été observée dans 259 communes réparties dans 44 départements. elle a été signalée à Aix-en-Provence. En 2012, des observations ont été réalisées dans six communes supplémentaires, localisées dans les Bouches-du-Rhône ainsi que dans quatre départements proches (Alpes-de-HauteProvence, Ardèche, Hérault et Var). C'est à partir de 2014, soit six ans après la première observation, que le nombre d'observations a commencé à croître plus fortement (figure 4). Le décalage temporel entre l'introduction initiale et le début de la croissance rapide de la population et de l'expansion de l'aire de distribution est un phénomène communément observé chez les espèces introduites (SAKAI et al., 2001) et a également été signalé, dans le cas de M. sculpturalis, en Italie (RUZZIER et al., 2020) et en Autriche (LANNER et al., 2020b).

À l'issue de la saison de vol 2016, M. sculpturalis avait été observée dans 72 communes françaises réparties dans 21 départements. Les observations concernaient quasiexclusivement la zone géographique localisée au sud de Lyon et à l'est de Montpellier. Cependant, l'année 2016 avait été marquée par un net bon géographique des observations : on notait une importante progression vers l'ouest avec une première mention dans les Pyrénées-Atlantiques ainsi qu'un saut vers le nord avec une première mention en Saône-etLoire (LE FÉON et al., 2018).

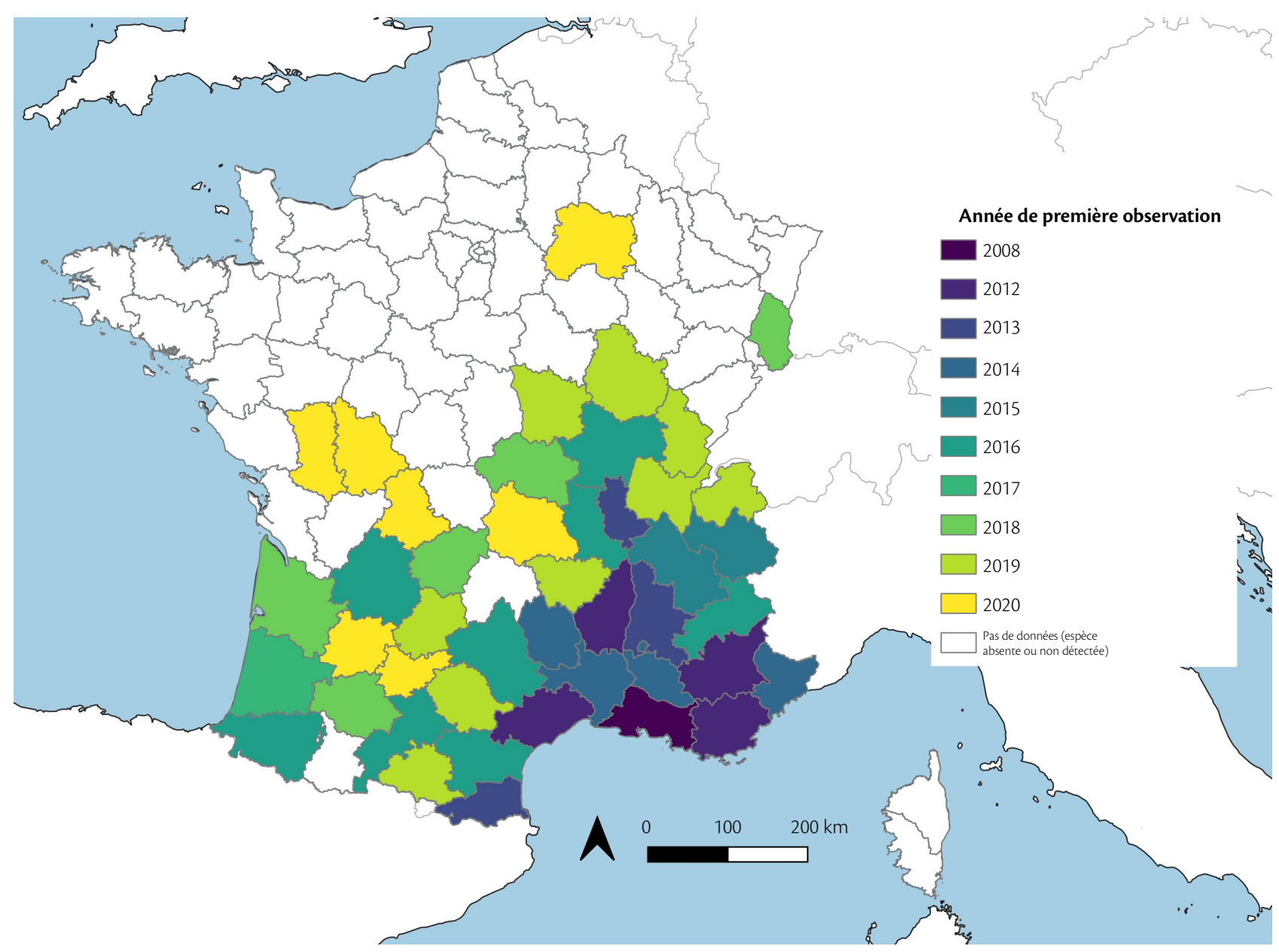

Figure 5. Année de première observation de Megachile sculpturalis dans les départements français.

Sources: LE FÉON et al., 2018 ; LE FÉON \& GESLIN, 2018 et témoignages directs ou observations collectées sur les plateformes et sites, principalement iNaturalist, INPN Espèces, Faune-France, Le Monde des Insectes, observation.org et Spipoll). Carte réalisée sous QGIS version 3.14, fond de carte téléchargé sur Natural Earth (naturalearthdata.com). Carte réalisée sous QGIS version 3.14, fond de carte téléchargé sur Natural Earth (https://www.naturalearthdata.com). 


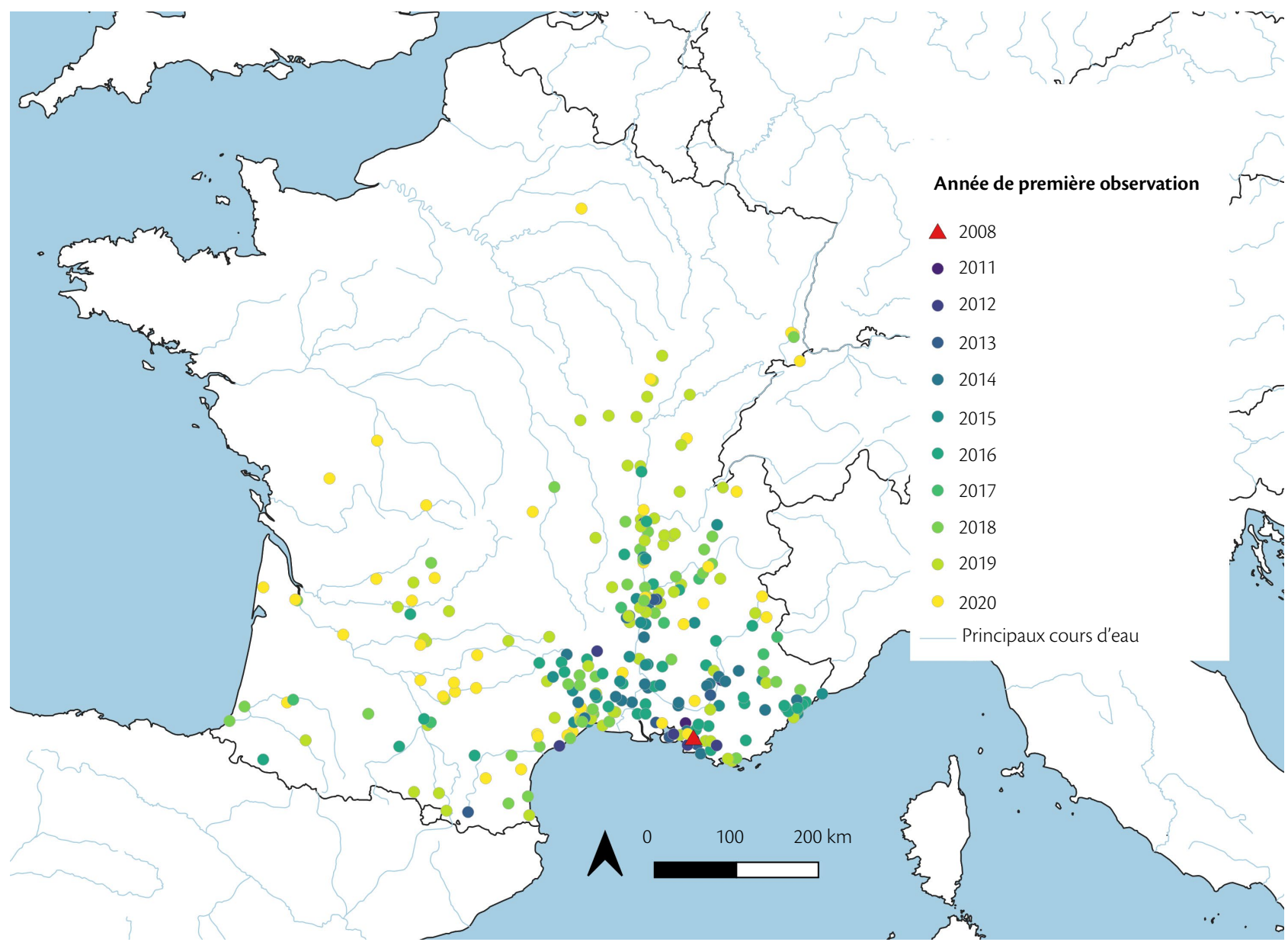

Figure 6. Distribution géographique de Megachile sculpturalis en France.

Chaque point représente une commune, la couleur indiquant l'année de première observation.

Sources: LE FÉON et al., 2018 ; LE FÉON \& GESLIN, 2018 et témoignages directs ou observations collectées sur les plateformes et sites, principalement iNaturalist, INPN Espèces, Faune-France, Le Monde des Insectes, observation.org et Spipoll). Carte réalisée sous QGIS version 3.14, fond de carte téléchargé sur Natural Earth (naturalearthdata.com). Carte réalisée sous QGIS version 3.14, fond de carte téléchargé sur Natural Earth (https://www.naturalearthdata.com).

À l'issue de la saison de vol 2017, M. sculpturalis avait été observée dans 77 communes françaises réparties dans 22 départements, avec une première mention pour le département des Landes, confirmant la progression vers l'ouest de l'espèce (LE FÉON \& GESLIN, 2018).

Qu'en est-il trois ans plus tard ? Nous avons collecté le maximum d'informations possible concernant les saisons de vol 2018, 2019 et 2020. En complément des sources d'informations utilisées précédemment et détaillées dans LE FÉON et al. (2018), nous avons également obtenu des données d'observation issues du portail naturaliste Faune France (https://www.faune-france.org), de l'application INPN Espèces (https://inpn.mnhn.fr/informations/inpnespeces) et de la plateforme internationale iNaturalist déjà mentionnée.

Afin de mobiliser le plus grand nombre d'observateurs, un appel à données a été lancé en juillet 2020 sur Faune France, sur la liste de diffusion Apoidea gallica et sur le site de l'Observatoire des Abeilles. Au niveau local, signalons l'action menée dès l'été 2019 par l'Observatoire de la faune de Bourgogne pour communiquer sur l'espèce et en savoir plus sur sa présence dans la région.

Il apparaît que ce sont dorénavant 259 communes réparties dans 44 départements qui comptent une ou plusieurs observations de M. sculpturalis (figures 5-6). Le quart Sud-Est concentre toujours la majorité des observations, Gard (22 communes citées), Hérault ( 21 communes) et Bouches-duRhône (19 communes) en tête.

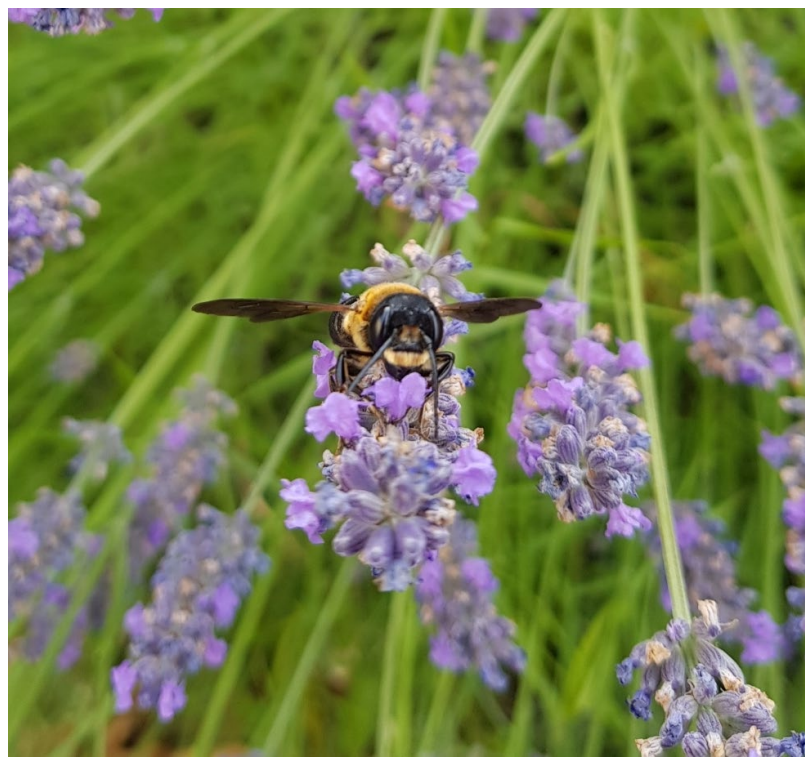

Figure 7. Mâle de Megachile sculpturalis butinant une fleur de lavande. Cette observation est la première ayant eu lieu au nord de Paris et constitue à l'heure actuelle l'observation la plus septentrionale en France. Noter la frange de poils clairs sur le devant de la tête, absente chez les femelles. Cliché B. Féry (juillet 2020, Vrigny, Marne, France). 
Dans 12 départements, l'espèce n'a été observée que dans une seule commune mais la progression vers l'ouest et le nord du pays se poursuit année après année. Fait marquant, la première observation au nord de Paris a eu lieu à Vrigny près de Reims (département de la Marne) le 9 juillet 2020. II s'agissait d'un mâle butinant une fleur de lavande (figure 7).

Megachile sculpturalis est observée aussi bien au cœur des grandes villes (Marseille bien sûr, mais aussi Bordeaux, Lyon, Montpellier ou Toulouse) que dans des zones rurales ou péri-urbaines ou des milieux naturels. En France, l'atitude maximale à laquelle elle a été observée reste 1846 mètres, donnée déjà mentionnée dans LE FÉON \& GESLIN (2018).

Nous avons collecté plusieurs témoignages mentionnant des concentrations d'individus (dont un témoignage vidéo). II s'agissait dans chaque cas de vieux arbres ou de troncs couchés offrant de nombreuses cavités dans lesquels plusieurs femelles (une dizaine à quelques dizaines) établissaient leur nid.
Enfin, une étude parue en 2020 (GESLIN et al., 2020) a permis de mieux évaluer la présence de l'espèce à Marseille, secteur probablement « berceau » de l'espèce en France. En février 2016, huit hôtels à abeilles, composés de troncs percés et de tiges de bambous ou de roseaux, ont été mis en place dans 12 parcs marseillais, soit un total de 96 hôtels. L'année suivante, les abeilles émergeant des troncs et des tiges ont été identifiées à l'espèce et comptabilisées. Au total, 889 abeilles sont sorties des hôtels, représentant cinq espèces : une espèce exotique, $M$. sculpturalis, et quatre espèces indigènes, Osmia bicornis, $O$. caerulescens, $O$. cornuta et $O$. niveata. Fait marquant, $M$. sculpturalis était l'espèce la plus abondante, représentant $40 \%$ des 889 abeilles ayant émergé, et a été trouvée dans 10 des 12 parcs étudiés. Cette étude a ainsi mis en évidence une présence forte de l'espèce dans l'ensemble de la ville, ceci seulement huit ans après sa découverte dans la commune voisine d'Allauch.

\section{PHÉNOLOGIE}

Megachile sculpturalis est univoltine. En France, sa saison de vol de s'étend de début juin à mi-septembre, avec un pic d'activité en juillet (LE FÉON et al., 2018) et c'est également globalement ce qui est observé dans les autres pays européens (BILA DUBAIĆ \& LANNER, 2021). Les mâles émergent avant les femelles.

\section{COMPORTEMENT ALIMENTAIRE}

Les données disponibles lors de notre précédente synthèse (LE FÉON \& GESLIN, 2018) indiquaient que M. sculpturalis visite de nombreuses espèces florales de diverses familles, à la fois d'origines exotique ou indigène. À l'échelle mondiale, elle a ainsi été observée sur les fleurs de 40 espèces végétales appartenant à 21 familles (PARYs et al., 2015). En France, entre 2008 et 2016, elle a été observée sur 20 espèces appartenant à 8 familles différentes (LE FÉON et al., 2018). Plusieurs publications récentes décrivent les observations de terrain réalisées dans différents pays d'Europe, ce qui amène à compléter la liste des plantes visitées. Par exemple, GUARIENTO et al. (2019) rapportent pour la première fois une activité de butinage sur le tournesol (Helianthus annuus), observation réitérée par RUZZIER et al. (2020). RUZZIER et al. (2020) rapportent pour la première fois du butinage sur Chamaerion angustifolium, Syringa sp. et Tetradium daniellii.

Chez les abeilles de façon générale, mâles et femelles visitent les fleurs pour la récolte du nectar pour leurs besoins propres. De plus, exception faite des abeilles-coucous, les femelles récoltent les ressources (pollen principalement) pour les larves. Le nombre de familles de plantes sur lequel une espèce d'abeille collecte du pollen détermine sa spécialisation alimentaire. Selon la classification simplifiée habituellement retenue (par exemple NIETO et al., 2014), on qualifie d'oligolectique une espèce collectant le pollen sur des plantes d'une seule famille et de polylectique une espèce collectant le pollen sur des plantes de plusieurs familles.

L'analyse du contenu pollinique des scopae des femelles ou des cellules larvaires est nécessaire pour connaître les espèces sur lesquelles le pollen est collecté et donc le statut de l'espèce, oligolectique ou polylectique. Nous décrivions, en 2018 , les analyses polliniques alors disponibles. QUARANTA et al. (2014), WESTRICH et al. (2015) et ANDRIEU-PONEL et al. (2018) avaient réalisé des analyses ponctuelles (un petit nombre de cellules larvaires ou scopae ayant été analysées dans chacune des études). Celles-ci indiquaient une prédominance du pollen de Sophora japonica (famille des Fabacées) et de troène (Ligustrum sp., famille des Oléacées). Du pollen de châtaignier était également présent. À notre connaissance, seuls AGUADO et al. (2018) ont depuis réalisé des analyses polliniques. À Sant Celoni (Catalogne, Espagne), les auteurs ont capturé cinq femelles pour analyser le pollen transporté. $100 \%$ du pollen transporté par quatre d'entre elles était du pollen de Sophora japonica. La cinquième transportait majoritairement du pollen de Sophora japonica mais également de petites quantités de pollen des genres Lagerstroemia (Lythracées) et Ligustrum. Sur un second site, à Olot (Catalogne, Espagne), les auteurs ont capturé une femelle. Le pollen transporté était cette fois constitué à $76 \%$ de Ligustrum vulgare (une espèce de troène naturellement présente en Europe), à $22 \%$ d'une autre espèce du genre Ligustrum, possiblement le troène exotique L. Iucidum présent sur le site, et à $2 \%$ de lavande (Lavandula sp., Lamiacées). Il s'agit une nouvelle fois d'une étude ponctuelle mais celle-ci tend à conforter les conclusions des études précédentes. II semble donc que $M$. sculpturalis soit une espèce polylectique avec une préférence marquée pour les espèces ligneuses exotiques originaires d'Asie (Sophora japonica et Ligustrum sp.) ou indigènes (Ligustrum vulgare). Des observations de terrain suggèrent que la glycine (Wisteria sinensis, Fabacées) et l'arbre à miel (Tetradium daniellii, Rutacées), deux arbustes également originaires d'Asie, sont utilisés par $M$. sculpturalis pour la récolte du pollen (respectivement GUARIENTO et al., 2019 et WESTRICH, 2020b), ce qui nécessite encore d'être validé par des analyses polliniques. 


\section{NIDIFICATION}

Incapable de creuser elles-mêmes une cavité, les femelles de M. sculpturalis utilisent diverses cavités préexistantes pour nidifier : tiges végétales comme les tiges de bambous présentes dans les hôtels à abeilles ou trous dans le bois. II peut s'agir de trous dans des arbres ou dans du bois de construction (poutres par exemples) ou encore de bûches percées placées dans les hôtels à insectes. Lors de leur étude dans les parcs marseillais, GESLIN et al. (2020) ont trouvé que le diamètre moyen des cavités utilisées par $M$. sculpturalis est de $9,5 \mathrm{~mm}$. La gamme de diamètres utilisée va de 5 à $15 \mathrm{~mm}$ environ (B. GESLIN, données non publiées). AGUADO et al. (2018) citent un nouveau matériau utilisé : les auteurs ont observé, en Espagne, une femelle nidifiant dans un trou d'un tube en plastique contenant un câble électrique. En août 2020, dans le Gard (France), plusieurs individus ont été observés autour de cavités situées dans le sol d'un talus sablonneux (figure 8). Ces cavités étaient, au moins pour certaines d'entre elles, d'anciens nids d'anthophores. Des individus ont à nouveau été observés autour des cavités dès la mi-juin 2021. En l'absence de l'observation de femelles transportant du pollen ou de la résine, il n'est pas possible d'indiquer avec certitude que ces cavités ont été utilisées pour nidifier mais des observations plus approfondies méritent sans doute d'être réalisées sur ce site.

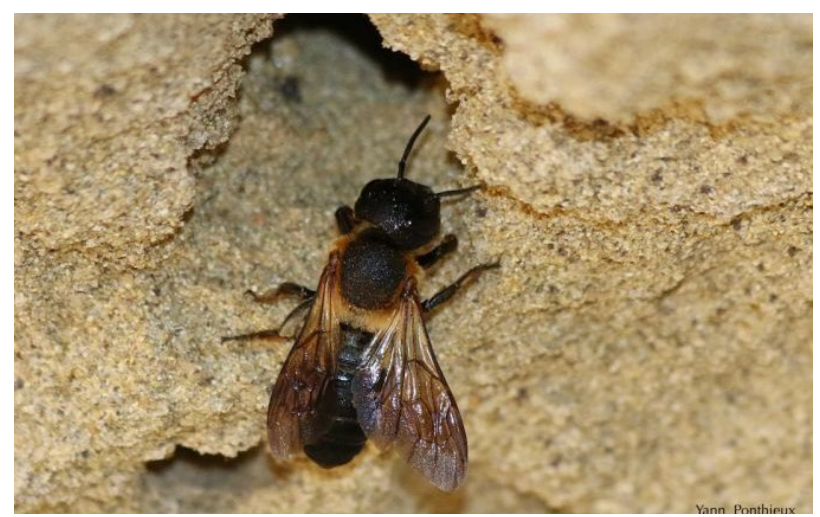

Figure 8. Femelle de Megachile sculpturalis sur un talus sablonneux. Cliché Y. PONTHIEUX (août 2020, Beauvoisin, Gard, France).

Les cellules larvaires sont construites en résine de conifères ou d'érable (BATRA, 1998). AGUADO et al. (2018) relatent l'observation de plusieurs femelles collectant de la résine sur un pêcher (Prunus persica).

Nous rapportions l'utilisation de trous anciennement occupés par des xylocopes dans plusieurs communes françaises (LE FÉON \& GESLIN, 2018). Le même type d'observations a été réalisé en Autriche (LANNER et al., 2020a) ou en Espagne (AGUADO et al., 2018). AGUADO et al. (2018) signalent également la nidification sur le même site d'Anthidium florentinum et de $M$. sculpturalis, sans qu'aucune interaction agressive n'ait été observée.

Nous signalions également l'observation de femelles de M. sculpturalis vidant le contenu de cellules larvaires d'osmies (Osmia cornuta et $O$. bicornis) dans deux communes françaises et l'observation d'une femelle vidant le contenu d'un nid de la guêpe Isodontia mexicana en Autriche (LE FÉON \& GESLIN, 2018). Cette tendance de M. sculpturalis à vider les nids d'autres espèces a été confirmée par de nouvelles observations. Ainsi, en Autriche et en Suisse, quatre observateurs ont signalé des nids d'O. cornuta vidés par des femelles de M. sculpturalis (LANNER et al., 2020a). Des photographies et vidéos qui nous ont été communiquées suggèrent également ce type de comportement sur des nids d'espèces du genre Anthidium (figure 9), notamment Anthidium septemspinosum (observations réalisées à Anglet dans les PyrénéesAtlantiques en 2018 et 2019). Enfin, signalons que ce comportement existe aussi dans son aire d'origine : des photographies montrent en effet une femelle de M. sculpturalis vidant le nid, rempli de chenilles, d'une guêpe solitaire à Pékin (Chine) $)^{4}$.

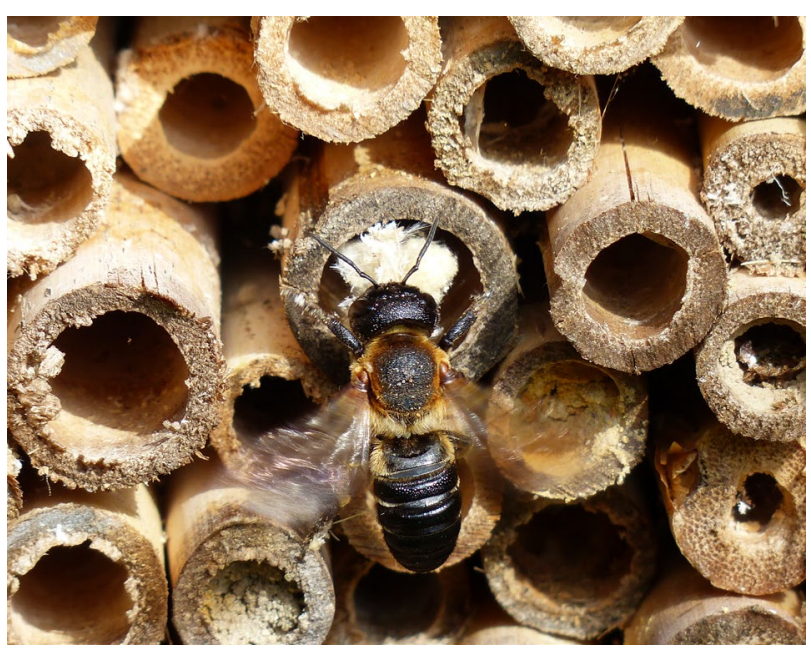

Figure 9. Femelle de Megachile sculpturalis vidant les poils végétaux emmagasinés dans un tube de bambou par une abeille du genre Anthidium. Cliché P.-J. VANDOORNE (juillet 2018, Anglet, PyrénéesAtlantiques, France)

Une étude scientifique récemment parue permet d'en apprendre un peu plus sur ce comportement (DíAz et al., 2021). En janvier 2018, une équipe de scientifiques italiens a placé, dans deux communes au sud de Turin, quatre hôtels à abeilles en panneaux de fibre de bois (deux dans chaque commune) constitués chacun de 81 tunnels de $10 \mathrm{~mm}$ de diamètre. En novembre 2018, ils ont ouvert les tunnels pour analyser leur contenu. Sur les 324 tunnels de l'expérimentation, 229 étaient occupés par des abeilles. Seules deux espèces ont été recensées, $O$. cornuta et M. sculpturalis. 171 tunnels (75\% des tunnels occupés) hébergeaient des larves d'O. cornuta, 58 tunnels ( $25 \%$ ) des larves de $M$. sculpturalis. Fait marquant, $44 \%$ des tunnels occupés par M. sculpturalis comportaient des cellules larvaires d'O. cornuta dans la partie arrière (c'est-à-dire éloignée de l'entrée). Cela suggère que, dans près de la moitié des cas, les femelles de $M$. sculpturalis ont vidé partiellement des nids d'O. cornuta (cette espèce étant printanière, les nids en question avaient été mis en place bien plus tôt dans la saison) et y ont ensuite installé leur nid, alors même que des tunnels non occupés étaient encore disponibles à proximité.

${ }^{4}$ https://www.flickr.com/photos/96008771@N03/36819653500/in/album-72157679813282223 (consultation juin 2021) 


\section{IMPACTS ÉCOLOGIQUES}

En Europe, M. sculpturalis est la première espèce d'abeille introduite à s'être largement répandue à travers le continent. Une autre espèce, $M$. disjunctiformis, également originaire de l'est de l'Asie, a été observée en Italie dès 2011 mais sa présence semble pour l'instant cantonnée à l'agglomération de Bologne (BORTOLOTTI et al., 2018 ; F. LUTHI, comm. pers. juin 2020). Dans plusieurs régions du monde, notamment sur le continent américain et en Océanie, les espèces d'abeilles exotiques sont nombreuses (GOULSON, 2003 ; RUsSO, 2016). Par exemple, BARTOMEUs et al. (2013) en ont recensé 20 espèces dans le nord-est des États-Unis. La présence d'abeilles hors de leur aire de répartition d'origine résulte souvent d'introductions involontaires associées au transport de marchandises. Parmi les introductions volontaires, celle de l'abeille mellifère (Apis mellifera), introduite pour la pollinisation des cultures et la production de miel, est la plus connue. D'autres espèces, telles que Bombus terrestris ou Megachile rotundata, sont aujourd'hui présentes hors de leur aire d'origine du fait de leur utilisation pour la pollinisation (GESLIN et al., 2017). D'après GOULSON (2003), les impacts négatifs associés à l'introduction d'abeilles exotiques peuvent être classés en cinq catégories : (1) la compétition pour les ressources florales avec les pollinisateurs indigènes; (2) la compétition pour les sites de nidification ; (3) l'introduction conjointe de pathogènes ; (4) la pollinisation de plantes exotiques et (5) les effets négatifs sur la reproduction des plantes indigènes.
Pour M. sculpturalis, les observations de terrain et les études portent jusqu'à présent principalement sur la problématique de la compétition pour les sites de nidification et de la tendance de l'espèce à vider les nids d'autres espèces. Dès 2012 aux États-Unis, LAPORT \& MINCKLEY (2012) et ROULSTON \& MALFI (2012) signalaient l'observation de femelles de M. sculpturalis chassant de leur nid des femelles de Xylocopa virginica puis vidant le contenu de la cavité afin d'y installer leurs propres cellules larvaires. Depuis, en Europe, l'installation de nids de M. sculpturalis dans des cavités précédemment utilisées par des abeilles du genre Xylocopa a été observée à de nombreuses reprises mais sans mention d'usurpation de nid en activité (voir toutefois l'observation d'une possible cohabitation relatée dans LE FÉON \& GESLIN, 2018). Par ailleurs, des femelles ont été observées vidant les nids d'autres espèces d'abeilles, en particulier Osmia cornuta, ou ceux de guêpes solitaires. L'expérimentation de DíAZ et al. (2021) a récemment permis de quantifier ce phénomène, dans le cas de quatre hôtels à abeilles installés près de Turin en Italie. Près de la moitié des nids de $M$. sculpturalis étaient installés dans des tunnels également occupés par $O$. cornuta, ce qui suggère que le comportement d'éviction a été fréquent alors même que des cavités non occupées étaient disponibles à proximité. Les auteurs de l'étude suggèrent que les impacts sur les populations d'O. cornuta sont de deux types. D'une part, l'éviction des larves ou des nymphes induit un effet direct

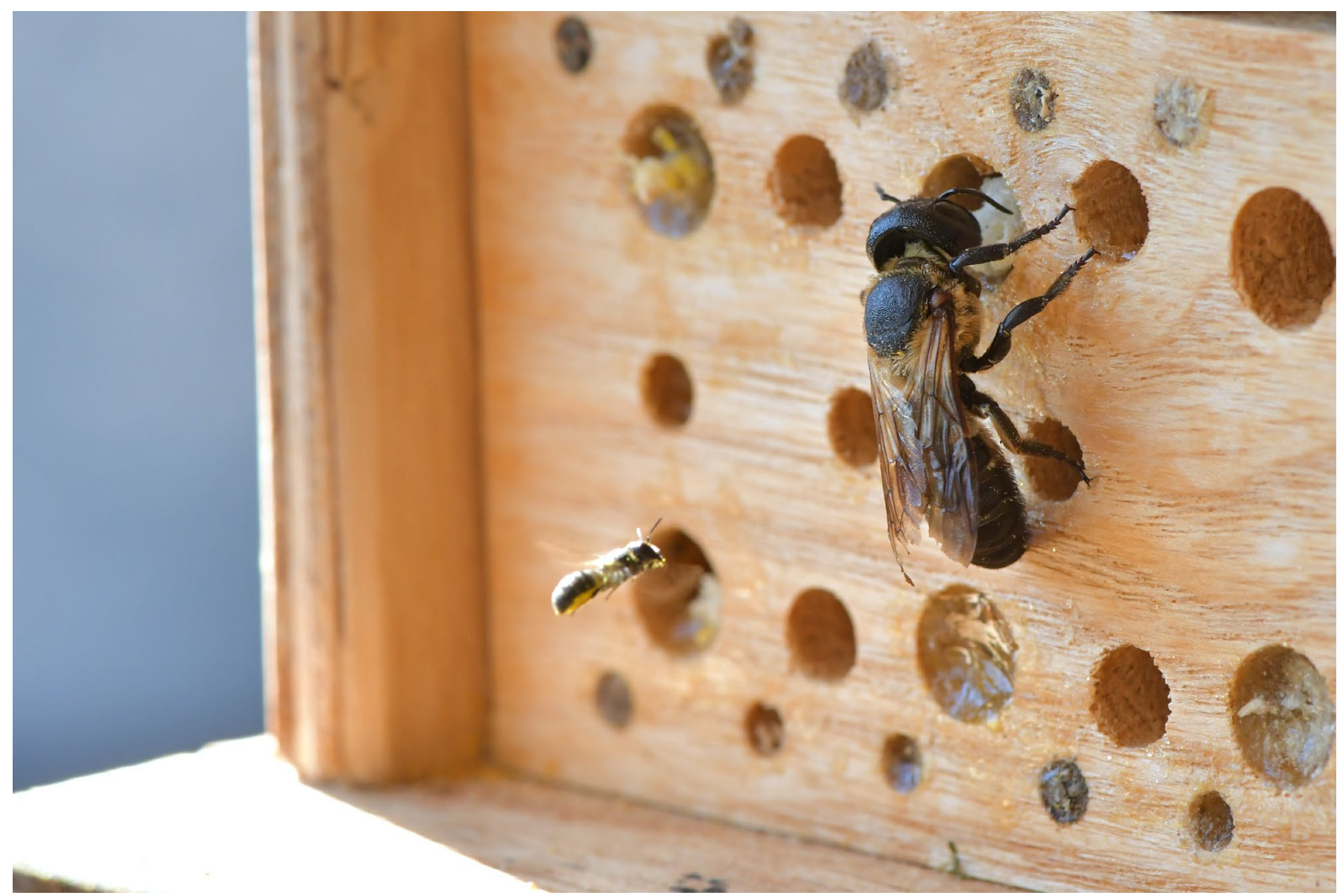

Figure 10. Femelle de Megachile sculpturalis en train de fermer son nid avec de la résine. À gauche, une femelle du genre Heriades rentre à son nid la brosse ventrale chargée de pollen. C'est sur ce site de nidification qu'une femelle a été observée tuant une femelle d'Heriades sp. Cliché C. ROHRBACH (août 2020, Oberhofen am Thunersee, Berne, Suisse). 
(mortalité des individus du fait de leur explusion du nid). D'autre part, la présence des cellules larvaires de $M$. sculpturalis devant celles des osmies induirait une mortalité indirecte en bloquant la sortie des adultes au printemps suivant, ceux-ci étant incapables de traverser les différentes cellules larvaires construites en résine par M. sculpturalis (DíAz et al., 2021).

En 2020, un nouveau type de comportement a été observé : une femelle de $M$. sculpturalis a tué une abeille du genre Heriades sur un site de nidification en Suisse (figure 10 et photographies présentées dans LANNER et al., 2020b). L'observatrice ayant photographié la scène écrit : « la femelle de M. sculpturalis a tué une abeille du genre Heriades et en a attaqué d'autres pendant que je l'observais. Les femelles $d$ 'Heriades $s p$. volaient souvent de la résine dans la réserve de résine de M. sculpturalis et dans les bouchons des nids. À ma grande surprise, elles n'évitaient pas M. sculpturalis lorsqu'elle revenait de sa recherche de nourriture. Souvent, M. sculpturalis enjambait simplement les voleuses lorsqu'elle entrait dans son nid, mais parfois elle en attrapait une, la jetait sur le côté ou même la tuait, comme cela a été observé dans un cas. " (ROHRBACH, 2020).
En l'état actuel des connaissances, il est difficile d'évaluer la fréquence de ces phénomènes et de quantifier l'impact que M. sculpturalis pourrait avoir sur les populations d'abeilles cavicoles indigènes. BILA DUBAIĆ \& LANNER (2021) suggèrent également de s'intéresser à la problématique de la compétition pour les ressources florales avec les pollinisateurs indigènes, en particulier en milieux urbains. Dans ces milieux en effet, les plantes visitées préférentiellement par $M$. sculpturalis constituent aussi des ressources alimentaires primordiales pour de nombreux insectes pollinisateurs pendant la période estivale.

L'arrivée de M. sculpturalis sur l'île d'Elbe (RUZZIER et al., 2020) et à Majorque (RIBAS-MARQUĖs \& DÍAZ-CALAFAT, 2021) pose également la question de la vulnérabilité particulière des écosystèmes insulaires vis-à-vis de l'introduction d'espèces exotiques (SIMBERLOFF, 1995 ; WHITTAKER \& FERNÁNDEZ-PALACIOS, 2007). RUZZIER et al. (2020) suggèrent d'ailleurs d'agir rapidement pour éviter une colonisation importante de l'île d'Elbe, voire d'autres îles de l'archipel toscan. Ils proposent d'utiliser des hôtels à abeilles pour localiser la présence de $M$. sculpturalis sur l'île puis de détruire les nids pour contenir l'accroissement des populations et éviter une propagation trop importante de l'espèce sur l'île.

\section{HÔTELS À ABEILLES : RECOMMANDATIONS POUR ÉVITER L'INSTALLATION DE MEGACHILE SCULPTURALIS}

Comme on vient de le voir, RUZZIER et al. (2020) proposent d'utiliser les hôtels à abeilles pour lutter contre la propagation de M. sculpturalis sur l'île d'Elbe. Dans la majorité des cas bien sûr, l'installation d'hôtels a pour objectif premier de fournir des sites de nidification à la faune indigène. On l'a vu, M. sculpturalis utilise massivement les hôtels pour nidifier. Comment faire, alors, pour qu'une action mise en place avec l'objectif de favoriser les abeilles indigènes ne fasse contre-emploi en favorisant l'expansion d'une espèce exotique potentiellement néfaste ? Megachile sculpturalis étant une abeille de grande taille, elle utilise pour nidifier des trous plus grands que la majorité des abeilles cavicoles de la faune française. Aussi, pour ne pas favoriser son implantation via l'installation des hôtels à abeilles, il est utile que ceux-ci comportent uniquement des cavités d'un diamètre inférieur à $8 \mathrm{~mm}$ (GESLIN et al., 2020). Une gamme de diamètres compris entre 4 et $8 \mathrm{~mm}$ permet d'héberger les principales espèces susceptibles de nidifier dans les hôtels à abeilles (à l'exception toutefois de certaines grandes espèces comme dans le genre Anthidium).

\section{APPEL À DONNÉES ET RECHERCHE DE SPÉCIMENS}

À l'heure où paraissent ces lignes, la saison de vol de M. sculpturalis bat son plein. Pour suivre de la façon la plus précise possible sa distribution géographique en France, approfondir les connaissances sur son écologie et mieux évaluer ses impacts sur la biodiversité, nous vous invitons à nous communiquer vos données (violette.lefeon@ gmail.com ; benoitgeslin@gmail.com). L'idéal est de prendre des photos et de noter le maximum d'informations : date, lieu (coordonnées géographiques si possible, sinon au minimum département, commune et rue ou lieu-dit dans le cas des grandes villes), nombre et sexe des spécimens, plantes visitées, substrats utilisés pour la nidification, interactions éventuelles avec d'autres abeilles.
Pour rechercher la présence de $M$. sculpturalis sur un territoire, sont à surveiller en particulier les plants de Sophora japonica, de troène et de lavande ainsi que les hôtels à abeilles, les vieux arbres et leurs abords.

Par ailleurs, l'acquisition de spécimens de $M$. sculpturalis est indispensable dans le cadre de nombreux projets, notamment les études de génétique et de morphométrie. Nous sommes de ce fait à la recherche de spécimens en provenance des différentes régions de France. Si vous avez la possibilité de collecter des spécimens, vous pouvez les stocker dans de l'alcool (éthanol $70^{\circ}$ ) et nous écrire. Les frais postaux pour l'acheminement vous seront remboursés. 


\section{REMERCIEMENTS}

Nous remercions vivement toutes les personnes qui nous communiquent leurs observations ou les partagent sur les plateformes naturalistes ainsi que les photographes qui nous ont permis d'illustrer cet article : Benoît FÉRY, Yann PONTHIEUX, Géraud DE Premorel, Christa RohrBACH, Pierre-Jean VANDOORNE et Frank VASSEN. Merci à Pierre-Jean VANDOORNE pour l'abondante documentation photo et vidéo qu'il nous a transmise et à Matthieu AUBERT pour avoir accepté de visionner une de ces vidéos afin de mettre un nom sur l'espèce d'anthidie que l'on y observe. Merci également à Matthieu AUBERT et l'Observatoire des Abeilles, Cécile DÉTROIT (Société d'histoire naturelle d'Autun), Benoit MARTHA, Quentin ROME et
Bertrand SCHATZ pour leur aide dans la collecte des informations. Les données issues du portail naturaliste Faune France sont intégrées à ce travail dans le cadre du partenariat, coordonné par David GENOUD, entre Faune France et l'Observatoire des Abeilles. Les données récentes du Spipoll ont été obtenues avec l'aide de Grégoire Loïs. Le suivi de M. sculpturalis en France a bénéficié en 2020 et 2021 d'un financement du GDR Pollinéco coordonné par Bertrand SCHATZ (groupe de recherche POLLINisation, réseaux d'interaction et fonctionnalité des ÉCOsystèmes). Enfin, nous remercions chaleureusement Tanguy JEAN et Adrien PERRARD pour la relecture de cet article, Tanguy JEAN ayant aussi assuré la mise en page. 


\section{RÉFÉRENCES}

Aguado, O., C. Hernández-Castellano, E. Bassols, M. Miralles, D. Navarro, C. Stefanescu \& N. Vicens (2018). Megachile (Callomegachile) sculpturalis SMITH, 1853 (Apoidea: Megachilidae): a new exotic species in the Iberian Peninsula, and some notes about its biology. Butlletí de la Institució Catalana d'Història Natural, 82: 157-162.

https://www.researchgate.net/publication/329170771_Megachil e_Callomegachile_sculpturalis_Smith_1853_Apoidea_Megachil idae_a_new_exotic_species_in_the_lberian_Peninsula_and_so me_notes_about_its_biology [accessed 15 June 2021]

AMIET, F. (2012). Die Blattschneiderbiene Megachile sculpturalis SMITH, 1853 (Hymenoptera, Apidae) nun auch in der Schweiz. Entomo Helvetica, 5: 157-159.

https://portal-cdn.scnat.ch/asset/f0369680-f0eb-5736-8a58-

3300e5404c4c/157_159_Amiet_Megachile?b=35ba46b7-0cd5-53d9a694-efb82bf70a20\&v =6879f101-22f6-5c84-bcca-

Odd90fd35e6b 0\&s =SEuBi4R-

LOpMrLYi76wTR9CtjRGsfZmbv9uWpYKwcrkoBdn_SIVr2tRyHpViojFABfsH7AfE4wF9ThYAiiakH3xXBpLS6YfZ33OHddcTOmN4AtyoySKkZ nEJ7cH7cotrPphF-gVPtllCO825bmpjSZC5dlZ7VvM3oPCc-xYrCY [accessed 15 June 2021]

Andrieu-Ponel, V., P. Ponel, V. Le Féon, B. Geslin \& G. Duvallet (2018). A propos du comportement de butinage de Megachile sculpturalis SMITH, 1853, en France méditerranéenne (Nîmes et Montpellier) (Hymenoptera, Megachilidae). Bulletin de la Société entomologique de France, 123(1): 49-54.

https://doi.org/10.32475/bsef_1984

Bartomeus, I, J. S. Ascher, J. GibBS, B. N. DANForth, D. L. WAGner, S. M. HEDTKE \& R. WINFREE (2013). Historical changes in northeastern US bee pollinators related to shared ecological traits. Proceedings of the National Academy of Sciences, 110(12): 4656-4660 https://doi.org/10.1073/pnas.1218503110

Batra S. W. T. (1998). Biology of the Giant Resin Bee, Megachile sculpturalis SMITH, a conspicuous new immigrant in Maryland. The Maryland Naturalist, 42(1-2): 1-3.

https://www.biodiversitylibrary.org/item/239237\#page/1/mode/ 1 up [accessed 15 June 2021]

BILA Dubaić J. B. \& J. LANner (2021). Megachile sculpturalis (Hymenoptera: Megachilidae): a valuable study organism for invasive pollinators and the role of beekeepers in ongoing monitoring programs. Bee World, 98(3): 5 pp. (unknown pagination at the date of consult). https://doi.org/10.1080/0005772X.2021.1940580

BORTOLOTTI, L., F. LUTHI, S. FlAMINIO, G. BOGO \& F. SGOLASTRA (2018). First record of the Asiatic bee Megachile disjunctiformis in Europe. Bulletin of Insectology, 71(1): 143-149.

http://www.bulletinofinsectology.org/pdfarticles/vol71-2018143-149bortolotti.pdf

Ćetrović, A., L. Stanisavljević, M. Pleća, J. Raičević, V. Žikić, M. Glavendekić \& J. Bila Dubaić (2020). Project: Monitoring the spread of sculptured resin bee (Megachile sculpturalis) in Serbia. Centar za biologiju pčela, Biološki fakultet, Univerzitet u Beogradu, Belgrade (Serbia).

https://sites.google.com/bio.bg.ac.rs/srbee/english [accessed 01 June 2021]

Díaz, S. S., L. Carisio, A. Manino, P. Biella, M. Porporato (2021). Nesting, sex ratio and natural enemies of the Giant Resin Bee in relation to native species in Europe. Insects, 12(6): 545. https://doi.org/10.3390/insects12060545

Geslin, B., B. Gauzens, M. Baude, I. Dajoz, C. Fontaine, M. Henry, L. Ropars, O. Rollin, E. Thébault \& N. J. Vereecken (2017). Massively introduced managed species and their consequences for pantpollinator interactions. Advances in Ecological Research, 57: 147199. https://doi.org/10.1016/bs.aecr.2016.10.007

Geslin, B., S. Gachet, M. Deschamps-Cottin, F. Flacher, B. Ignace, C. KNoploch, E. MeIneri, C. Robles, L. Ropars, L. SChURR \& V. Le FÉON (2020). Bee hotels host a high abundance of exotic bees in an urban context. Acta Oecologica, 105: 103556 https://doi.org/10.1016/j.actao.2020.103556
GogalA, A. \& B. ZADRAVEC (2018). First record of Megachile sculpturalis Smith in Slovenia (Hymenoptera: Megachilidae). Acta Entomologica Slovenica, 26(1): 79-82.

http://www.pms-lj.si/si/files/default/Publikacije/Strokovnaglasila/Acta-entomologica-slovenica/2018/1/GogalaZadravec_AES\%2026-1_2018.pdf [accessed 15 June 2021]

GoulsON, D. (2003). Effects of introduced bees on native ecosystems. Annual Review of Ecology, Evolution and Systematics, 34: 1-26. https://doi.org/10.1146/annurev.ecolsys.34.011802.132355

Guariento, E., J. Lanner, M. A. Staggl \& P. Kranebitter (2019). Megachile sculpturalis (SMITH, 1853) (Hymenoptera: Megachilidae), the giant resin bee new to South Tyrol with a newly described plant species interaction. Gredleriana, 19: 209215. http://doi.org/10.5281/zenodo.3565365

IVANOV, S. P. \& A. V. FATERYGA (2019). First record of the invasive giant resin bee Megachile (Callomegachile) sculpturalis SMITH, 1853 (Hymenoptera: Megachilidae) in the Crimea. Far Eastern Entomologist, 395: 7-13. https://doi.org/10.25221/fee.395.2

KováCS, T. (2015). Megachile sculpturalis SMITH, 1853 in Hungary (Hymenoptera, Megachilidae). Folia Historico-naturalia Musei Matraensis, 39: 73-76.

https://matramuzeum.nhmus.hu/sites/default/files/nhmusfiles/k iadvanyok/folia/vol39/08_Megachile_Kovacs_39.pdf [accessed 15 June 2021]

Lanner, J., K. Huchler, B. PAChinger, C. Sedivy \& H. MeimberG (2020a). Dispersal patterns of an introduced wild bee, Megachile sculpturalis SMITH, 1853 (Hymenoptera: Megachilidae) in European alpine countries. PLOS ONE, 15(7): e0236042. https://doi.org/10.1371/journal.pone.0236042

LANNER, J., P. Meyer, F. HARMetZKY, H. MeimberG \& B. PACHinger (2020b). Die Asiatische Mörtelbiene (Hymenoptera: Megachile sculpturalis SMITH, 1853) - eine neue Bienenart für Österreich. Beiträge zur Entomofaunistik, 21: 87-95.

https://www.researchgate.net/publication/347460952_Die_Asiat ische_Mortelbiene_Hymeno_ptera_Megachile_sculpturalis_Sm ith_1853_-eine_neue_Bienenart_fur_Osterreich [accessed 15 June 2021]

LanNer, J., F. Gstöttenmayer, M. Curto, B. Geslin, K. Huchler, M. C. OrR, B. Pachinger, C. Sedivy \& H. MeimberG (2021). Evidence for multiple introductions of an invasive wild bee species currently under rapid range expansion in Europe. BMC Ecology and Evolution, 21: 17. https://doi.org/10.1186/s12862-020-01729-x

LAPORT, R. G. \& R. L. MINCKLEy (2012). Occupation of active Xylocopa virginica nests by the recently invasive Megachile sculpturalis in Upstate New York. Journal of the Kansas Entomological Society, 85(4): 384-386. https://doi.org/10.2317/0022-8567-85.4.384

Le FÉON, V., M. Aubert, D. Genoud D., V. AndRIEu-POnel, P. Westrich \& B. GESLIN (2018). Range expansion of the Asian native giant resin bee Megachile sculpturalis (Hymenoptera, Apoidea, Megachilidae) in France. Ecology and Evolution, 8(3): 1534-1542. https://doi.org/10.1002/ece3.3758

LE FÉON, V. \& B. GEsLIN (2018). Écologie et distribution de l'abeille originaire d'Asie Megachile sculpturalis Smith 1853 (Apoidea Megachilidae - Megachilini) : un état des connaissances dix ans après sa première observation en Europe. Osmia, 7: 31-39. https://doi.org/10.47446/OSMIA7.6

MANGUM, W. A. \& R. W. BROOKS (1997). First records of Megachile (Callomegachile) sculpturalis SMITH (Hymenoptera: Megachilidae) in the continental United States. Journal of the Kansas Entomological Society, 70: 140-142. https://www.jstor.org/stable/25085766

Nieto, A., S. P. M. Roberts, J. Kemp, P. Rasmont, M. Kuhlmann, M. García Criado, J. C. Biesmelier, P. Bogusch, H. H. Dathe, P. De la Rúa, T. De Meulemeester, M. Dehon, A. Dewulf, F. J. Ortiz-Sánchez, P. lhomme, A. Pauly, S. G. Potts, C. Praz, M. Quaranta, V. G. RadChenko, E. SCheuchl, J. SMit, J. Straka, M. Terzo, B. Tomozil, J. WINDOW \& D. MICHEZ (2014). European red list of bees. 
Luxembourg, Publication office of the European Union. https://doi.org/10.2779/77003

Ortiz-Sánchez, F. J., J. F. Navarro, U. Taeger (2018). Megachile (Callomegachile) sculpturalis SMITH, 1853, nueva especie para la fauna ibérica (Hymenoptera, Megachilidae). Boletín de la Sociedad Entomológica Aragonesa, 63: 259-261.

ParYs, K., A. TrIPODI, B. Sampson (2015). The Giant Resin Bee, Megachile sculpturalis SMITH: New distributional records for the Mid- And Gulf-South USA. Biodiversity Data Journal, 3: e6733. https://doi.org/10.3897/BDJ.3.e6733

Quaranta, M., A. Sommaruga, P. Balzarini \& A. Felicioli (2014). A new species for the bee fauna of Italy: Megachile sculpturalis continues its colonization of Europe. Bulletin of Insectology, 67(2): 287-293. http://www.bulletinofinsectology.org/pdfarticles/vol67-2014287-293quaranta.pdf

Ribas-Marquès, E. \& J. Díaz-Calafat (2021). The Asian giant resin bee Megachile sculpturalis SMITH 1853 (Hymenoptera: Apoidea: Megachilidae), a new exotic species for the bee fauna of Mallorca (Balearic Islands, Spain). Journal of Apicultural Research, 60(3): 506-511. https://doi.org/10.1080/00218839.2021.1874177

ROHRBACH, C. (2020). Dangerous neighbourhood dispute. Flickr.

https://www.flickr.com/photos/megaptera/50287276353/in/datepos ted [accessed 21 June 2021].

Roulston, T \& R. MALFI (2012). Aggressive eviction of the Eastern Carpenter Bee (Xylocopa virginica (LINNAEUS)) from its nest by the Giant Resin Bee (Megachile sculpturalis SMITH). Journal of the Kansas Entomological Society, 85(4): 387-388. https://doi.org/10.2317/0022-8567-85.4.387

Russo, L. (2016). Positive and negative impacts of non-native bee species around the world. Insects, 7(4): 69. https://doi.org/10.3390/insects7040069

Ruzzier, E., M. Menchetti, L. Bortolotti, M. Selis, E. Monterastelli \& L. FORBICIONI (2020). Updated distribution of the invasive Megachile sculpturalis (Hymenoptera: Megachilidae) in Italy and its first record on a Mediterranean island. Biodiversity Data Journal, 8 : e57783. https://doi.org/10.3897/BDJ.8.e57783
SAKAI, A. K., F. W. Allendorf, J. S. Holt, D. M. LOdGe, J. MOlofsky, K. A. With, S. Baughman, R. J. Cabin, J. E. Cohen, N. C. Ellstrand, D. E. McCauley, P. O’Nell, I. M. Parker, J. N. Thompson \& S. G. Weller (2001). The population biology of invasive species. Annual Review of Ecology and Systematics, 32: 305-332.

https://doi.org/10.1146/annurev.ecolsys.32.081501.114037

SIMBERLOFF, D. (1995). Why do introduced species appear to devastate islands more than mainland areas? Pacific Science, 49(1), 87-97. http://hdl.handle.net/10125/2276

VereECKEN, N. J. \& É. BARBier (2009). Premières données sur la présence de l'abeille asiatique Megachile (Callomegachile) sculpturalis SMITH (Hymenoptera, Megachilidae) en Europe. Osmia, 3: 4-6. https://doi.org/10.47446/OSMIA3.3

Westrich, P., A. KNAPp \& I. Berney (2015). Megachile sculpturalis SMITH 1853 (Hymenoptera, Apidae), a new species for the bee fauna of Germany, now north of the Alps. Eucera, 9: 3-10. https://www.zobodat.at/pdf/Eucera_9_2015_0001-0010.pdf [accessed 15 June 2021]

WESTRICH, P. (2017). Faszination Wildbienen, Forschungsprojekte: Megachile sculpturalis. wildbienen.info. https://www.wildbienen.info/forschung/projekte_17.php [accessed 01 June 2021]

WESTRICH, P. (2020a). Faszination Wildbienen, Forschungsprojekte: Megachile sculpturalis. [document Internet]. wildbienen.info. https://www.wildbienen.info/forschung/projekte_20.php [accessed 01 June 2021]

Westrich, P. (2020b). Neues zur Ausbreitung der Mörtelbiene Megachile sculpturalis SMITH 1853 (Hymenoptera: Anthophila) in Deutschland - Stand Oktober 2019. Eucera, 14: 12-15. https://www.zobodat.at/pdf/Eucera_14_2020_0012-0015.pdf [accessed 15 June 2021]

WHITTAKER, R.J. \&J. M. FERNÁNDEZ-PALACIOS (2007). Island biogeography: Ecology, evolution, and conservation. Second edition. Oxford University Press, Oxford (UK), 416 pp.

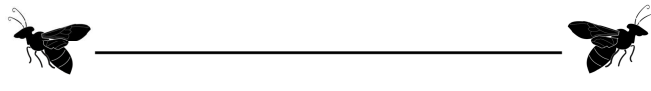

OSMIA est éditée par l'Observatoire des Abeilles (OA), une association loi 1901 d'apidologues (ou mellitologues) d'Europe francophone qui œuvrent pour la connaissance et la protection des Abeilles sauvages.

Les articles sont:

- publiés uniquement en ligne

- publiés uniquement en ligne

- disponibles en open access.

Crossref Zoobank, HAL, Zenodo, OpenAIRE, Google Scholar et Web of Science (Clarivate) [Zoological Record],

respectueux des recommandations de la Commissio internationale de Nomenclature zoologique (ICZN)

- sous Licence Creative Commons Attribution International CC BY 4.0 qui autorise la reproduction et la diffusion du document, à condition d'en citer explicitement la source

- librement déposables sur des sites internet ou des plateformes d'archivage.

(!) Les documents d'autres sources et non distribuées sous licence libre sont reproduits après autorisation (à demander par les auteurs) et demeurent la propriété des auteurs ou éditeurs originaux. originaux.
(!) Le contenu publié est sous l'entière responsabilité des

OSMIA est conçue pour une impression recto-verso en haute résolution. Les bibliothèques publiques, les laboratoires, les muséums et les associations sont invités à imprimer et conserver une version papier de la revue.
OSMIA is published by the Observatory of Bees (OA), a nonprofit society of apidologists (or mellitologists) from Frenchspeaking Europe who work together for the knowledge and protection of wild bees

The items are

published only online

avallable in open access, OpenAIRE, Google Scholar and Web of Science (Clarivate) OpenAIRE, Google Scho
[Zoological Record],

[Zoological Record],

- respectful of the recommendations of the Inte
Commission for Zoological Nomenclature (ICZN),

under Creative Commons Attribution Licence International CC BY 4.0 which authorises the reproduction and distribution of the document, provided the source is explicitly cited,

- freely depositable on personal or institutional websites and archiving platforms.

(!) Documents from other sources and not distributed under a free license are reproduced after authorisation (to be requested by the authors) and remain the property of the original authors or publishers

(!) The published content is the sole responsibility of the
or publishers. authors.

OSMIA is designed for high-resolution printing on both sides. Public libraries, laboratories, museums, and societies are invited to print and keep a paper version of the journal.

\section{Directeur de la publication • Editor-in-chief Benoît GESLIN}

Comité éditorial • Editorial Board Matthieu AUBERT • Floriane FLACHER • Mehdi ISSERTES . Tanguy JEAN • Léa LEMAIRE

Mise en page $\cdot$ Layout

Mehdi ISSERTES • TanguY IEAN • Léa LEMAIRE

Comité de lecture • Scientific committee 2021 https://www.osmia-journal-hymenoptera.com/equipe-team.html.

Soumission d'articles • Submission of items osmia.editor@gmail.com

Recommandations aux auteurs

Recommendations to authors

https:/ /www.osmia-journal-hymenoptera.com/auteurs-authorsinstructions.html

Observatoire des Abeilles 68 rue du Onze Novembre F - 59148 Flines-lez-Râches (France) https://oabeilles.net/

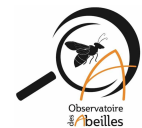

open ACCESS 